\title{
The Use of Suture Augmentation for Graft Protection in ACL Reconstruction: A Biomechanical Study in Porcine Knees
}

\author{
Vince J. Lai, M.D., Alan W. Reynolds, M.D., Michael Kindya, M.D., John Konicek, B.S., and \\ Sam Akhavan, M.D.
}

\begin{abstract}
Purpose: To biomechanically evaluate the use of the suture augmentation construct at time 0 of ACL reconstruction. Methods: Eighty porcine knees underwent ACL reconstruction using 2 techniques for graft fixation: a single suspensory construct (SSC), performed with a femoral button and tibial interference screw; and a double suspensory construct (DSC), with a femoral and tibial button. Each fixation technique was performed on 40 porcine knees divided into 4 subgroups. The first group had a nonaugmented ACL reconstruction, the second group had an ACL reconstruction with suture augmentation, and the third and fourth groups were the same as the first and second groups, with the graft resected $80 \%$ to simulate graft weakening. Ultimate load, yield load, stiffness, cyclic displacement values, and mode of failure were recorded for each graft. Results: In a weakened graft model with $80 \%$ graft resection, there was a significant increase in ultimate strength $(P<.001)$, yield strength $(P<.001)$, and cyclic displacement $(P<.001)$ with suture augmentation. There was no significant increase in stiffness with suture augmentation with either construct $(P=.278)$. In the setting of an intact graft, there were no differences in either SSC or DCS groups with or without suture augmentation. Conclusions: The addition of a suture to ACL reconstruction techniques resulted in minimal changes in baseline biomechanical characteristics while improving ultimate load, yield load, and cyclic displacement in a weakened graft model. Clinical Relevance: Suture augmentation of ACL reconstruction may confer improved integrity of the graft and is worth consideration and future clinical study.
\end{abstract}

A nterior cruciate ligament (ACL) injuries have an incidence of 200,000 annually in the United States, ${ }^{1}$ making ACL reconstruction one of the most common ligament reconstruction procedures. The percentage of ACL reconstructions requiring revision surgeries is relatively high, $\sim 10 \%$ to $15 \% .^{2-6}$ Prior research from the Multicenter ACL Revision Study

From the Department of Orthopaedic Surgery, Allegheny Health Network, Pittsburgh, Pennsylvania, U.S.A. (V.J.L., A.W.R., S.A.); Orthopaedic Associates of Osceola, Kissimmee, Florida, U.S.A. (M.K.); and Arthrex, Inc., Naples, Florida, U.S.A. (J.K.).

The authors report the following potential conflicts of interest or sources of funding: J.K. reports other, Arthrex. S.A. reports other, Arthrex. Full ICMJE author disclosure forms are available for this article online, as supplementary material.

Received June 1, 2020; accepted August 22, 2020.

Address correspondence to Alan W. Reynolds, M.D. Department of Orthopedic Surgery, Allegheny General Hospital, 320 E North Ave, Pittsburgh, PA, 15212,U.S.A.E-mail: alanwwreynolds@gmail.com

(C) 2020 THE AUTHORS. Published by Elsevier Inc. on behalf of the Arthroscopy Association of North America. This is an open access article under the CC BY-NC-ND license (http://creativecommons.org/licenses/by-nc-nd/4.0/). 2666-061X/20973

https://doi.org/10.1016/j.asmr.2020.08.009
(MARS) Group has shown the factors leading to ACL revision reconstructions to include trauma (32\%), technical factors $(24 \%)$, or biologic factors $(7 \%) .{ }^{7}$ One concern that may contribute to failure of the graft is trauma to the graft during the initial phases of ligamentization.

The ligamentization process can be prolonged, lasting $\leq 6$ months until its final stages. ${ }^{8}$ During this time, the graft will undergo extensive remodeling, with a significant decrease in its mechanical properties. ${ }^{9-13}$ The ultimate strength of the graft during these phases may decrease by as much as $80 \%$ of its original strength, putting the graft at risk for plastic deformation or failure. ${ }^{6,9,12}$

Suture augmentation of ACL reconstructions has been used to increase the strength of the graft construct during the early revascularization and remodeling phase. ${ }^{14.15}$ Cook et al. ${ }^{16}$ looked at using synthetic augmentation for ACL reconstructions in a live canine model and concluded that the biologicsynthetic load-sharing ACL construct prevented early failure and allowed for functional graft remodeling while avoiding problems with use of all-synthetic 


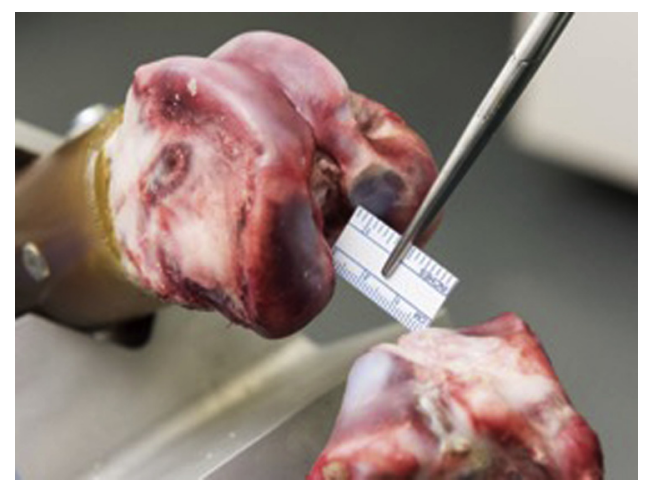

Fig 1. Demonstration of measurement of the intra-articular anterior cruciate ligament (ACL) length, as was performed on all porcine knees. The dissected specimen in this image includes the distal femur on the left of the image and proximal tibia in the bottom right.

grafts. Furthermore, concerns exist that suture augmentation may stress shield the graft during the initial phases of healing.

The purpose of this study was to biomechanically evaluate the use of the suture augmentation construct at time 0 of ACL reconstruction. It was hypothesized that the use of suture augmentation would be protective to the graft, especially in the setting of a compromised graft.

\section{Methods}

\section{Specimen Preparation}

We performed ACL reconstruction using 2 commonly used constructs in porcine knee specimens. Proximal femurs and distal tibias were potted in fiberglass resin. Our first model was a single suspensory construct (SSC) using button fixation in the femur and an interference screw for fixation in the tibia. Our second model was a double suspensory construct (DSC) using button fixation in both the femur and tibia. All porcine knees were stripped of all soft tissues before performing the ACL reconstructions.

\section{Femoral Tunnel and Intra-Articular Length Determination}

In both constructs, the femoral tunnel was made as a 20-mm socket using an outside-guide and Flip-Cutter drill (Arthrex, Naples, FL). We ensured that femoral intraosseous length was $\geq 30 \mathrm{~mm}$ to ensure a stout cortical wall for button fixation. Femoral fixation was performed using a TightRope button (Arthrex). The intra-articular graft length was determined by fixing the porcine leg in independent femoral and tibial clamps and using a ruler to make sure the tunnels were $30 \mathrm{~mm}$ apart. Demonstration of this intra-articular measurement is shown in Figure 1.

\section{Tibial Tunnel}

In the SCC group, the full tibial tunnel was drilled using a guide wire followed by a reamer. The graft was passed and fixed with a line-to-line Biocomposite interference screw (Arthrex).

In the DSC group, a socket was drilled using a flip cutter drill. The socket was drilled to the step bullet on the guide, ensuring a 7-mm bone bridge for the TightRope button. This ensured that the socket would be long enough to allow for graft tensioning. Once the graft was secured on both the femoral and tibial side, the graft was then cycled through flexion-extension motion and retightened, and the sutures on the button were tied to secure the loop.

\section{Graft Preparation}

For the SSC group, we used a quadrupled bovine extensor tendon construct. The ends of each graft were sutured using 2-0 FiberWire (Arthrex). The graft was passed through the loop of the TightRope RT button, while ensuring equal length of the graft limbs.

For the DSC group, a GraftLink (Arthrex) construct was made using bovine extensor tendon. The graft was mounted with TightRope RT button for the femur and a TightRope ABS for the tibia.

\section{Suture Augmentation}

In cases in which suture augmentation was used, FiberTape suture (Arthrex) was passed through the femoral button, ensuring equal length of sutures on each side of the graft. In the SSC model, the suture was brought through the cannulation of the tibial interference screw. Once the graft was fixed on the femoral and tibial side, the FiberTape suture was tensioned separately and fixed on the tibia with a $4.75-\mathrm{mm}$

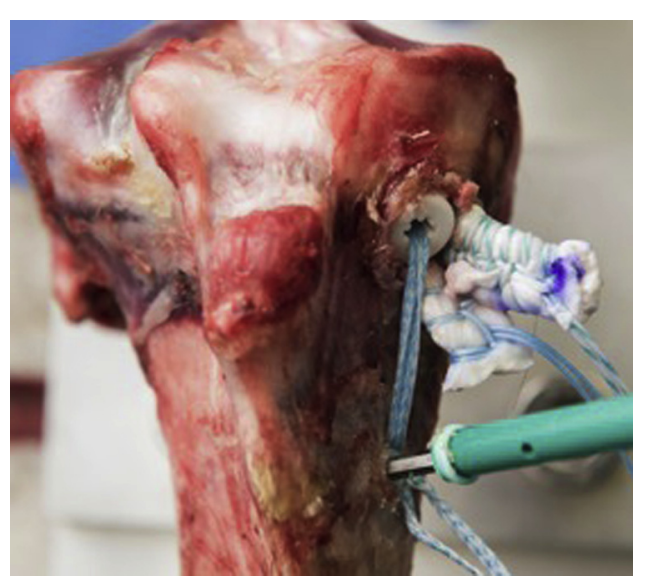

Fig 2. Demonstration of the tibial fixation in a single suspensory construct, in which fixation was achieved with an interference screw and the suture augmentation with a more distally placed anchor. In this image, the entire proximal tibia has been dissected, with the fixation and tunnel on the medial side. 


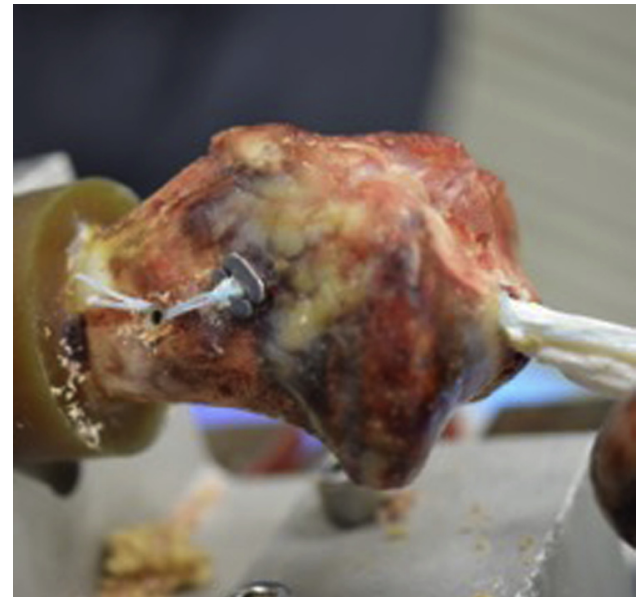

Fig 3. Demonstration of tibial fixation in a double suspensory construct using a suture button. In this image, the tibia shaft is on the left of the image with the plateau on the right; it is viewed from the medial side.

SwiveLock anchor (Arthrex). Demonstration of tibia fixation is shown in Figure 2 for this group.

In the DSC model, the suture was tensioned separately and fixed on the tibia with a 4.75-mm SwiveLock anchor after the graft was tensioned and fixed on the femur and tibia. This technique of tibial fixation can be seen in Figure 3. A view of the complete reconstruction is shown in Figure 4.

\section{Graft Resection}

For the groups undergoing graft resection, an $80 \%$ graft resection of each individual limb of the graft was performed. The limbs of the graft were measured with a digital caliper (Mitutoyo, Aurora, IL), and $80 \%$ of its diameter was resected using a scalpel. This resection technique is shown in Figure 5. Prior studies in the literature have shown that the ACL graft has significantly diminished strength properties in the postoperative stages. The amount of graft resection was chosen to allow for a definitive decrease in graft strength, beyond what has been shown in the literature. $6,10,13,16$

\section{Group Allocation}

A total of 80 porcine knees were tested, with 40 specimens allocated to each model. For each model, we divided the 40 specimens into 4 groups of 10 specimens each.

The first group underwent a standard SSC or DSC reconstruction. The second group underwent a SSC or DSC reconstruction with a Fibertape suture augmentation. The third group underwent a SSC or DSC reconstruction with $80 \%$ resection, and the fourth group underwent a SSC or DSC reconstruction with $80 \%$ resection and Fibertape suture augmentation.

\section{Specimen Testing}

All specimens were mounted on a materials testing machine (E10000; Instron Corp., Norwood, MA) with the femur held vertically to the cross-head and the tibia held at $30^{\circ}$ flexion to the testing surface. Samples were preconditioned for 10 sinusoidal cycles, at 10 to $50 \mathrm{~N}$ and $1 \mathrm{~Hz}$. Sinusoidal cyclic loading of the samples was conducted for 500 cycles, at 50 to $250 \mathrm{~N}$ and $1 \mathrm{~Hz}$, followed by a single cycle load to failure at $20 \mathrm{~mm} / \mathrm{min}$. Load and displacement data were recorded at $1000 \mathrm{~Hz}$. Plastic cyclic displacement, stiffness, yield load, and ultimate load were measured directly from the load-displacement curves. Stiffness was measured from the slope of the linear portion of the load-displacement curve immediately after cyclic loading.

\section{Power and Statistical Analysis}

A post hoc power analysis of the ultimate load comparisons was performed using SigmaPlot version 11.0 (Systat Software, San Jose, CA). For $\alpha=0.05$, the power of the two-way ANOVA was 1.000, for both the presence of an internal brace and graft condition. Furthermore, the interaction between the presence of an internal brace and the condition of the graft had a power of .856 .

Statistical analysis was performed using two-way ANOVA, with $P<.05$ considered significant. Analysis of failure modes was performed using a Fisher exact test, with $P<.05$ considered significant.

\section{Results}

\section{Single Suspensory Construct}

Intact Graft Versus Intact Graft With Suture
Augmentation

In the SSC model, augmentation with a suture did not result in a significant increase from baseline in

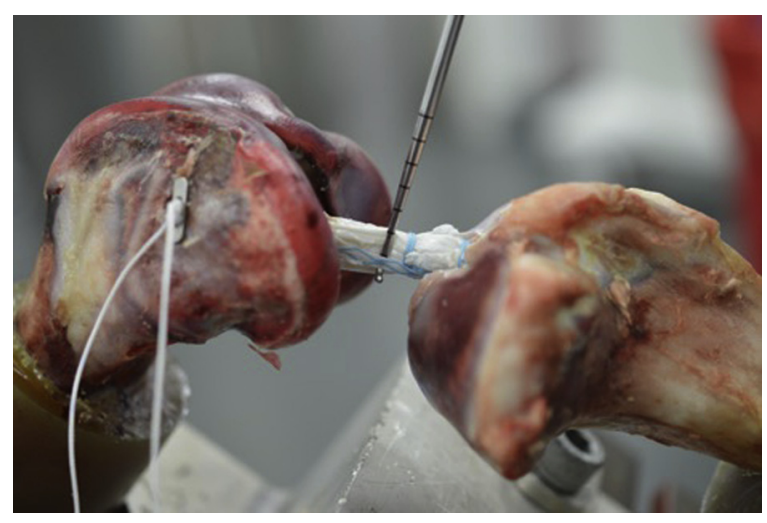

Fig 4. The completed soft tissue anterior cruciate ligament (ACL) reconstruction with addition of suture augmentation. In this image as viewed from the lateral side, the distal femur is at the left of the image. 


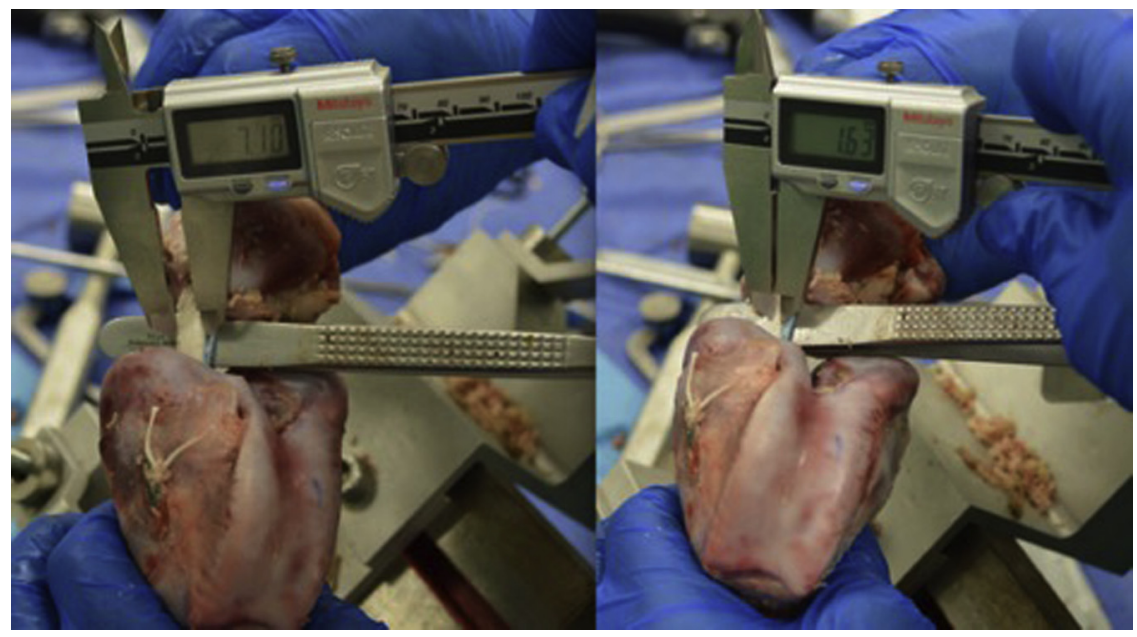

Fig 5. Demonstration of soft tissue anterior cruciate ligament (ACL) reconstruction undergoing measured resection to $80 \%$ of width. In the left image, the width of the graft is measured with calipers after reconstruction is complete. As shown in the image on the right, $80 \%$ of the width was then resected for this group. In these images, the distal femur is at the bottom of the image and the knee is slightly flexed.

biomechanical properties. There was no significant difference in ultimate strength $(892 \pm 116$ versus 1000 $\pm 139 \mathrm{~N} ; P=.057)$, yield strength $(826 \pm 122$ versus $820 \pm 186 \mathrm{~N} ; P=.927)$, or cyclic displacement $(5.3 \pm$ 1.1 versus $4.4 \pm 0.7 \mathrm{~mm} ; P=.057)$. There was no significant difference in stiffness when a suture augmentation was performed ( $146 \pm 28$ versus $139 \pm$ $28 \mathrm{~N} / \mathrm{mm} ; P=.172$ ).

\section{Resected Graft Versus Resected Graft With Suture Augmentation}

When the graft was resected $80 \%$, there was a significant difference in ultimate load $(263 \pm 82$ versus $655 \pm 149 \mathrm{~N} ; P<.001)$, yield strength $(263 \pm 82$ versus $623 \pm 124 \mathrm{~N} ; P<.001)$, and cyclic displacement $(9.5 \pm$ 1.6 versus $6.0 \pm 1.0 \mathrm{~mm} ; P<.001)$. There was no significant difference in stiffness when a suture augmentation was performed $(85 \pm 20$ versus $121 \pm 9$ $\mathrm{N} / \mathrm{mm} ; P=.148)$.

\section{Intact Graft Versus Resected Graft With Suture Augmentation}

Adding a suture augmentation to an $80 \%$ resected graft did not fully restore the biomechanical properties of the construct in the SCC model. There was a significant difference in terms of ultimate strength (891 \pm 116 versus $655 \pm 149 \mathrm{~N}, P<.001$ ), yield strength
$(826 \pm 122$ versus $623 \pm 124 \mathrm{~N}, P=.002)$, and cyclic displacement $(5.3 \pm 1.1$ versus $6.0 \pm 1.0 \mathrm{~mm}, P=.001)$ compared with an intact state. There was no significant difference in terms of stiffness ( $146 \pm 28$ versus $121 \pm 9$ $\mathrm{N} / \mathrm{mm}$ ). Single suspensory results are summarized in Table 1 .

\section{Mode of Failure}

With an intact graft and no suture augmentation, in $80 \%$ of the cases, the mode of failure was the graft slipping past the interference screw. These results are summarized in Table 2 . With the addition of suture augmentation, in $80 \%$ of cases, the mode of failure was the button breaking through the cortex. This finding was statistically significant $(P=.023)$.

With a resected graft and no suture augmentation, in $90 \%$ of cases, the failure mode was failure and stretching of the graft. With the addition of suture augmentation, in $80 \%$ of cases, the mode of failure was failure and stretching of the graft. This finding was not significant $(P=1.0)$.

\section{Double Suspensory Construct}

Intact Graft Versus Intact Graft With Suture Augmentation

In the DSC model, augmentation with a suture resulted in no significant difference in ultimate

Table 1. Single Suspensory Construct Data and Comparisons

\begin{tabular}{|c|c|c|c|c|c|c|c|}
\hline \multirow[b]{2}{*}{ Factor } & \multirow{2}{*}{$\begin{array}{l}\text { Intact Graft } \\
\text { Only }(a)\end{array}$} & \multirow{2}{*}{$\begin{array}{l}\text { Intact Graft Suture } \\
\text { Augmentation }(b)\end{array}$} & \multirow{2}{*}{$\begin{array}{c}\text { Graft } 80 \% \\
\text { Resected }(c)\end{array}$} & \multirow{2}{*}{$\begin{array}{c}\text { Graft } 80 \% \text { Resected } \\
\text { With Augmentation }(d)\end{array}$} & \multicolumn{3}{|c|}{$P$ Value } \\
\hline & & & & & $a$ vs. $b$ & $c$ vs. $d$ & $a$ vs. $d$ \\
\hline Ultimate strength $(\mathrm{N})$ & $891 \pm 116$ & $1000 \pm 139$ & $263 \pm 82$ & $655 \pm 149$ & .057 & $<.001$ & $<.001$ \\
\hline Yield strength $(\mathrm{N})$ & $826 \pm 122$ & $820 \pm 186$ & $263 \pm 82$ & $623 \pm 124$ & .927 & $<.001$ & .005 \\
\hline $\begin{array}{l}\text { Cyclic displacement } \\
\quad(\mathrm{mm})\end{array}$ & $5.3 \pm 1.1$ & $4.4 \pm 0.7$ & $9.5 \pm 1.6$ & $6 \pm 1.0$ & .111 & $<.001$ & .117 \\
\hline Stiffness (N/mm) & $146 \pm 28$ & $139 \pm 28$ & $85 \pm 20$ & $121 \pm 9$ & .428 & .148 & .09 \\
\hline
\end{tabular}

Data are mean \pm standard deviation. 
Table 2. Single Suspensory Construct Mode of Failure

\begin{tabular}{lcccc}
\hline \multicolumn{1}{c}{ Failure Mode } & $\begin{array}{c}\text { Intact Graft, } \\
\text { No Suture }\end{array}$ & $\begin{array}{c}\text { Intact Graft, Suture } \\
\text { Augmentation }\end{array}$ & $\begin{array}{c}\text { Resected Graft, } \\
\text { No Suture }\end{array}$ & Resected Graft, Suture Augmentation \\
\hline $\begin{array}{l}\text { Graft slippage past screw } \\
\begin{array}{c}\text { Button breaking/button } \\
\text { pulled through bone }\end{array}\end{array}$ & 8 & 2 & 1 & 1 \\
$\begin{array}{l}\text { Graft failure } \\
\text { Data are } \mathrm{n}\end{array}$ & 0 & 8 & 0 & 1 \\
\hline
\end{tabular}

strength, yield strength, or cyclic displacement. There was no significant difference in stiffness with suture augmentation.

\section{Resected Graft Versus Resected Graft With Suture Augmentation}

When the graft was resected $80 \%$, there was a significant difference in ultimate strength, yield strength, and cyclic displacement with suture augmentation. There was no significant difference in stiffness when a suture augmentation was performed.

\section{Intact Graft Versus Resected Graft With Suture Augmentation}

Adding a suture augmentation to an $80 \%$ resected graft fully restored the biomechanical properties of the construct in the DSC model. There was no significant difference in terms of ultimate strength, yield strength, and cyclic displacement compared with an intact state. There was also no significant difference in terms of stiffness. Double suspensory results are summarized in Table 3.

\section{Mode of Failure}

With an intact graft and no suture augmentation, in $100 \%$ of the cases, the mode of failure was button breakage on the tibia. With the addition of suture augmentation, in $90 \%$ of cases, the mode of failure was the button breaking on the tibia. This finding was not statistically significant $(P=1.0)$. Listing of these findings can be found in Table 4.

With a resected graft and no suture augmentation, in $100 \%$ of cases, the failure mode was failure and stretching of the GraftLink construct. With the addition of suture augmentation, in $50 \%$ of cases, the mode of failure was failure and stretching of the Graftlink. In the remaining $50 \%$, the failure was the button breaking on the tibia. This finding was statistically significant $(P=$ $.033)$.

\section{Discussion}

The results of the current study demonstrate the biomechanical protective effect of suture augmentation in the setting of ACL reconstruction, especially in a weakened graft. This "weakened state" can be seen during the ligamentization phase of ACL reconstruction, which can last $\geq 6$ months after surgery. $3,6,17$

The process of ligamentization has been well described in the literature and includes early-healing, proliferative, and maturation phases. Progression through these stages will include graft necrosis, revascularization, cellular repopulation, deposition of collagen fibers, and remodeling. $2,9,12,17-20$ When the graft is in the early healing stages ( 3 to 8 weeks postoperatively), there are regions of central necrosis leading to graft weakening. ${ }^{8,18}$ The amount of central necrosis in the ACL graft is more evident in animal studies as opposed to the human studies. ${ }^{6,9-11,13} \mathrm{Hu}$ man ligamentization studies have shown a less robust process of graft necrosis and remodeling; the time frame, however, has been shown to extend longer than in an animal model. ${ }^{5,8,21,22}$

The reconstructions in the current study were performed using 2 commonly used reconstruction techniques. Our first model was a single-suspensory construct (SSC) model, using a femoral button and a tibial interference screw. When the graft was intact, suture augmentation of the graft did not result in significant increases of ultimate strength, yield strength,

Table 3. Double Suspensory Construct Data and Comparisons

\begin{tabular}{|c|c|c|c|c|c|c|c|}
\hline Factor & $\begin{array}{c}\text { Intact Graft } \\
\text { Only }(a)\end{array}$ & $\begin{array}{l}\text { Intact Graft Suture } \\
\text { Augmentation }(b)\end{array}$ & Graft $80 \%$ Resected $(c)$ & $\begin{array}{c}\text { Graft } 80 \% \\
\text { Resected With } \\
\text { Augmentation }(d)\end{array}$ & \multicolumn{3}{|c|}{$P$ Value } \\
\hline$\overline{\text { Ultimate strength }(\mathrm{N})}$ & $747 \pm 86$ & $850 \pm 216$ & $372 \pm 139$ & $798 \pm 116$ & .327 & $<.001$ & .441 \\
\hline Cyclic displacement (mm) & $5.2 \pm 1.9$ & $3.8 \pm 0.8$ & $8.9 \pm 3.3$ & $4.9 \pm 1.2$ & .286 & .001 & .735 \\
\hline Stiffness $(\mathrm{N} / \mathrm{mm})$ & $118 \pm 14$ & $128 \pm 22$ & $79 \pm 18$ & $108 \pm 20$ & .237 & .022 & .398 \\
\hline
\end{tabular}

\footnotetext{
Data are mean \pm standard deviation.
} 
Table 4. Double Suspensory Construct Mode of Failure

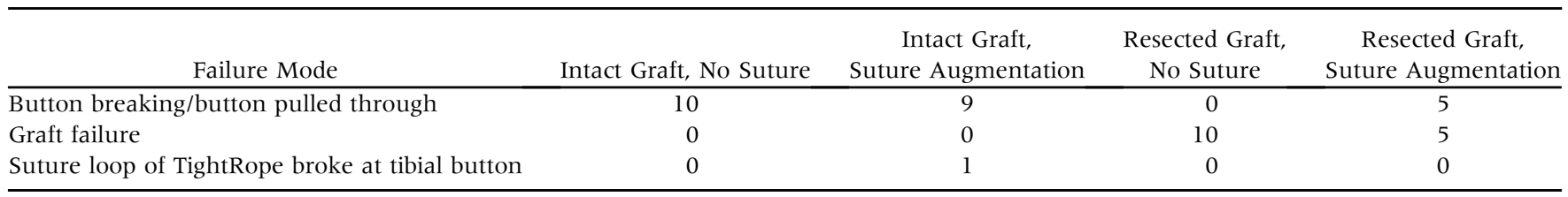

Data are $n$.

and cyclic displacement. Analysis of the mode of failure in suture augmentation with a tibial interference screw, however, demonstrated that suture augmentation may reduce graft slippage past the screw when the construct is exposed to tension forces.

Our second model was a double-suspensory construct (DSC) model, using a button for both femoral and tibial fixation. When the graft was intact, there was a no significant difference in the biomechanical properties when suture augmentation was used. The limiting factor for failure is the strength of the button in both graft conditions.

When the graft was resected, the addition of suture augmentation to both the SSC and DSC models significantly increased the biomechanical properties of the construct. In the setting of the DSC model, these properties were essentially normalized to the intact state. Most significantly, the suture augmentation significantly reduced the cyclic displacement of the construct, acting to protect the graft in the weakened state.

One of the biggest concerns with the use of suture augmentation is the potential for stress shielding of the graft. ${ }^{14,16}$ Our current study demonstrated that at initial implantation, the use of suture augmentation did not increase the overall stiffness of the construct, and thus theoretically should not lead to stress shielding of the graft. This is important, because stress shielding is a concern for any ACL augmentation because of delayed graft integration. ${ }^{23}$ We believe an important aspect to prevent stress shielding is to tension and fix the ACL graft independently from the suture augmentation. With independently and appropriately tensioned graft and augmentation, the graft will see the bulk of the stresses, with the suture augmentation becoming relevant only when the forces may overwhelm the graft. Theoretically, this should protect the ACL graft during its weakest stage, preventing plastic deformation of the graft and allowing the patient to proceed through the early phase of ligamentization with a biomechanically stronger reconstruction. ${ }^{14}$

\section{Limitations}

There are several limitations to the current study. First, this study used a cadaveric porcine model stripped of soft tissue attachments. The soft tissue envelope, notably the muscles surrounding the knee, can provide additional stability and strength to a graft despite the weakening during the ligamentization stage. Second, we simulated the weakened graft state through a simple resection of the graft, versus an intrinsic replacement of graft tissue by immature tissue starting with the peripheral moving centrally. Finally, our model only tested the construct at initial implantation and does not take into account the in vivo surroundings of the graft during the healing phases of ACL reconstruction. Further research should evaluate the clinical relevance and importance of use of a simple suture augment to protect the ACL reconstruction during the ligamentization phase in an in vivo model.

\section{Conclusions}

The addition of a suture to ACL reconstruction techniques resulted in minimal changes in baseline biomechanical characteristics while improving ultimate load, yield load, and cyclic displacement in a weakened graft model.

\section{Acknowledgments}

A portion of this work was supported by a research grant from Arthrex, Inc. (Naples, FL).

\section{References}

1. Samitier G, Marcano AI, Alentom Geli E, et al. Failure of anterior cruciate ligament reconstruction. Arch Bone Joint Surg 2015;3:220-240.

2. Arnoczky SP. Biology of ACL reconstructions: What happens to the graft? Instr Course Lect 1996;45:229-233.

3. Rougraff B, Shelbourne KD, Gerth PK, Warner J. Arthroscopic and histologic analysis of human patellar tendon autografts used for anterior cruciate ligament reconstruction. Am J Sports Med 1993;21:277-284.

4. Pauzenberger L, Syre S, Schurz M. 'Ligamentization' in hamstring tendon grafts after anterior cruciate ligament reconstruction: A systemic review of the literature and a glimpse into the future. Arthroscopy 2013;10:1712-1721.

5. Bachmann G, Cassens J, Heinrichs C, et al. MRT of the knee in the followup of the plastic repair of anterior cruciate ligament with autologous semitendinosus tendon. Rofo 1994;161:446-452 [in German].

6. Kondo E, Yasuda K, Katsura T, Hayashi R, Kotani Y, Tohyama H. Biomechanical and histological evaluations of the double semitendinosus tendon autograft after 
anterior cruciate ligament reconstruction in sheep. Am J Sports Med 2012;40:315-324.

7. Wright RW, Huston LJ, Spindler KP, et al. Descriptive epidemiology of the multicenter ACL revision study (MARS) cohort. Am J Sports Med 2010;38:1979-1986.

8. Janssen RPA, Scheffler SU. Intra-articular remodeling of hamstring tendon grafts after anterior cruciate ligament reconstruction. Knee Surg Sports Traumatol Arthrosc 2014;22:2102-2108.

9. Ballock RT, Woo SLY, Lyon RM, Hollis JM, Akeson WH. Use of patellar tendon autograft for anterior cruciate ligament reconstruction in the rabbit: A long-term histologic and biomechanical study. J Orthop Res 1989;7:474-485.

10. Grana WA, Egle DM, Mahnken R, Goodhard CW. An analysis of autograft fixation after anterior cruciate ligament reconstruction in a rabbit model. Am J Sports Med 1994;22:344-351.

11. Clancy WG, Narechania RG, Rosenberg TD, Gmeiner JG, Wisnefske DD, Lange TA. Anterior and posterior cruciate ligament reconstruction in rhesus monkeys. J Bone Joint Surg Am 1981;63:1270-1284.

12. Ng GY, Oakes BW, Deacon OW, et al. Biomechanics of patellar tendon autograft for reconstruction of the anterior cruciate ligament in the goat: Three-year study. J Orthop Res 1995; 13:602-608.

13. Papageorgiou CD, Ma B, Abramowitch SD, Clineff TD, Woo SLY. A multidisciplinary study of the healing of an intraarticular anterior cruciate ligament graft in a goat model. Am J Sports Med 2001;29:620-626.

14. Smith PA, Bley JA. Allograft anterior cruciate ligament reconstruction utilizing internal brace augmentation. Arthrosc Tech 2016;5:el143-el147.
15. Wilson WT, Hopper GP, Byrne PA, MacKay GM. Anterior cruciate ligament repair with internal brace ligament augmentation. Surg Technol Int 2016;29:273-278.

16. Cook JL, Smith PA, Stannard JP, Pfeiffer F, Kuroki K, Bozynski CC, Cook C. A canine arthroscopic anterior cruciate ligament reconstruction model for study of synthetic augmentation of tendon allografts. J Knee Surg 2017;30:704-711.

17. Claes S, Verdonk P, Forsyth R, Bellemans J. The 'ligamentization' process in anterior cruciate ligament reconstruction. Am J Sports Med 201 1;39:2476-2483.

18. Fu FH, Bennett $\mathrm{CH}$, Lattermann C, Ma B. Current trends in anterior cruciate ligament reconstruction Part l: Biology and biomechanics of reconstruction. Am J Sports Med 1999;27:821-830.

19. Weiler A, Peine R, Pashmineh-Azar A, Abel C, Sudkamp NP, Hoffmann RFG. Tendon healing in a bone tunnel. Part I: Biomechanical results after biodegradable interference fit fixation in a model of anterior cruciate ligament reconstruction in sheep. Arthroscopy 2002;18:1 13-123.

20. Armiel D, Kleiner JB, Roux RD, Harwood FL, Akeson WH. The phenomenon of 'ligamentization': Anterior cruciate ligament reconstruction with autogenous patellar tendon. J Orthop Res 1986;4:162-172.

21. Scheffler SU, Unterhauser FN, Weiler A. Graft remodeling and ligamentization after cruciate ligament reconstruction. Knee Surg Sports Traumatol Arthrosc 2008;16:834-842.

22. Zaffagnini $S$, et al. Neoligamentization process of BTPB used for ACL graft: Histological evaluation from 6 months to 10 years. Knee 2007; 14:87-93.

23. Herald J, Kaktakar S. Synthetic grafts in anterior cruciate ligament reconstruction. Asian J Arthrosc 2016;1:16-19. 